\title{
Eligibility Criteria for Patients Undergoing Allogeneic Hematopoietic Cell Transplantation
}

\author{
Abraham S. Kanate, MD'; Miguel-Angel Perales, MD²; and Mehdi Hamadani, MD,4
}

\section{ABSTRACT}

Eligibility assessment of a potential candidate for allogeneic hematopoietic cell transplantation (allo-HCT) is a complex yet vital component of pretransplant evaluation. Although no formal standardized consensus exists to guide this process, transplant centers follow institutional standard operating procedures and parameters to approve candidacy of an individual patient. Consideration for allo-HCT is dependent on a myriad of interrelated factors, including disease-related (eg, appropriate indication, disease status, prior therapies), patient-related (eg, age, functional status, frailty, comorbidities), psychosocial, and economic factors. A multidisciplinary approach is optimal for patient selection and requires the efforts of transplant coordinators, nurses, advanced practice providers, social workers, psychologists, financial specialists, and physicians. This article reviews the data and provides general guidelines that may be used in making an informed decision when evaluating a prospective candidate for allo-HCT. These recommendations are based on published data, expert commentary, reviews, and institutional practices. In the end, the eligibility assessment and decision to consider allo-HCT as the optimal choice of treatment for an individual patient are truly as much an "art" as it is the "science" of medicine, encompassing a multidisciplinary approach to minimize harm without compromising the curative potential-all essential doctrines of the Hippocratic Oath.

J Natl Compr Canc Netw 2020;18(5):635-643 doi: $10.6004 /$ jnccn.2020.7559

\footnotetext{
${ }^{1}$ Hematopoietic Malignancy and Cellular Therapy Program, West Virginia University, Morgantown, West Virginia; ${ }^{2}$ Adult Bone Marrow Transplantation Service, Memorial Sloan Kettering Cancer Center, New York, New York; and ${ }^{3}$ BMT \& Cellular Therapy Program, and ${ }^{4}$ Department of Medicine, Center for International Blood and Marrow Transplant Research, Medical College of Wisconsin, Milwaukee, Wisconsin.
}

Allogeneic hematopoietic cell transplantation (alloHCT) is a standard therapy option for the treatment of advanced hematologic malignancies and select nonmalignant disorders, with $>8,000$ transplants performed each year in the United States. ${ }^{1}$ Despite the curative potential of allo-HCT, the procedure is often associated with significant mortality risk, as well as short- and long-term morbidities such as graft-versus-host disease (GVHD), serious infections, organ toxicity, hemorrhage, second malignancies, and prolonged immunosuppression. In fact, an nonrelapse mortality (NRM) rate of $10 \%$ to $25 \%$ is often attributed to allo-HCT in the first 2 years and underlines the importance of appropriate evaluation and counseling of prospective transplant candidates. ${ }^{2}$ However, cohort data evaluating time trends show significant improvements in overall survival (OS) and NRM in recipients of allo-HCT over the years (2003-2007 vs 2013-2017), underlining the advancements made in HCT and supportive care procedures. ${ }^{3}$ In addition to providing accurate, easy to understand, and concise information regarding the pros and cons of the proposed procedure to aid in patient decisionmaking, the initial evaluations also serve to assess eligibility and suitability of allo-HCT as the optimal treatment for the individual patient. Providing educational written materials and videos and appropriate internet resources (eg https://bethematch.org, https:// www.cancer.gov) beforehand can empower the patient and family with correct information, thus making the initial consultation more meaningful and steering them clear of inaccurate web resources. ${ }^{2}$

Transplant evaluation, workup, patient counseling, and donor search take several weeks to coordinate, underscoring the need for early patient referral to transplant centers given that optimal timing of allo-HCT is an established determinant of outcomes. ${ }^{4}$ Although the underlying diagnosis invariably directs the initial transplant consultation, the final decision to proceed to allo-HCT is a complete process, dependent not just on disease-related factors (eg, remission status) but also on several patient-related factors, including physiologic, psychologic, social, and economic factors, as well as 
consideration of donor and graft source availability. The eligibility assessment of an individual patient to proceed to allo-HCT requires the concerted efforts and expertise of a team of transplant coordinators and nurses, advanced practice providers, research coordinators, social workers, psychologists, and financial specialists, in addition to experienced transplant physicians. Due to the lack of prospective data or formal consensus and guidelines for eligibility assessments for allo-HCT, this process varies significantly across transplant centers. Notably, nearly $90 \%$ of transplant centers in the United States are reviewed and accredited by the Foundation for the Accreditation of Cellular Therapy (FACT), thus establishing minimum standards for medical and laboratory practices. In Europe, the Joint Accreditation Committee ISCT-Europe \& European Society for Blood and Marrow Transplantation (EBMT; JACIE) provides accreditation. This article reviews the available data to provide an overview and general guidance for eligibility criteria for patients being evaluated for allo-HCT. Although these recommendations are largely based on published data, expert commentary, and reviews, it is inevitable that the authors' personal and institutional preferences are reflected here as well. ${ }^{5}$

\section{Disease-Related Factors}

Initial referral to the transplant center by the primary hematologist or oncologist for consideration of allo-HCT is invariably based on the underlying diagnosis. Over the past 3 to 4 decades, allo-HCT as a therapeutic strategy has indeed shifted from a last resort option to the treatment of choice in several instances. ${ }^{6}$ However, in the ever-changing field of hematology and oncology, it is imperative that the most recent and up-to-date information be reviewed to establish the suitability and timing of allo-HCT for a specific histologic diagnosis. It is vital to compare the risks and benefits of allo-HCT with other nontransplant treatment modalities and offer the best treatment option for the specific disease entity at that time for the individual patient. This has been clearly illustrated in examples such as chronic myeloid leukemia and, more recently, 17p-deleted chronic lymphocytic leukemia, in which oral tyrosine kinase inhibitors have supplanted allo-HCT as the primary frontline treatment of choice. The often-touted potential for cure with allo-HCT must not blind clinicians to the potentially significant risk of NRM.

\section{Indication for Allo-HCT}

The underlying disease is a key determinant to the initial consideration of allo-HCT for an individual patient. Table 1 summarizes indications for allo-HCT, including those in hematologic malignancies, nonmalignant hematologic disorders, and solid tumors, based on guidelines from the American Society for Transplantation and Cellular Therapy (ASTCT). ${ }^{6}$ These guidelines represent a comprehensive assessment of literature and include recommendations for standard of care and developmental indications, as well as recommendations regarding when transplant is not appropriate. For example, allo-HCT may be considered the standard of care for some diseases (eg, acute myeloid leukemia [AML] or myelodysplastic syndromes [MDS]), whereas in others it may be considered developmental and ideally offered in the context of a clinical trial (eg, solid tumors). The optimal timing for recommending allo-HCT in the disease course also plays an important role in patient outcomes $^{4}$ (Table 1). More comprehensive reviews of literature and guidelines for specific indications for alloHCT have been reported elsewhere but are beyond the scope of this article. ${ }^{6-8}$

\section{Prior Therapies}

Prior anticancer therapies may result in cumulative longterm adverse effects on performance scores and endorgan function, with important ramifications on many aspects of transplantation (eg, conditioning intensity selection, GVHD prophylaxis, veno-occlusive disease [VOD] risk). In patients with a history of prior autologous HCT, reduced-intensity conditioning/nonmyeloablative conditioning (RIC/NMAC) allo-HCT may be considered to mitigate the high risk of NRM associated with myeloablative conditioning in this setting. ${ }^{9}$ Similarly, therapies such as gemtuzumab ozogamicin for treatment of refractory AML, inotuzumab ozogamicin for acute lymphoblastic leukemia, or hepatic radiation may increase the subsequent risk of VOD with allo-HCT, which may prompt the use or avoidance of specific conditioning regimens or GVHD prophylaxis. ${ }^{10,11}$ Pretransplant exposure to mogamulizumab (CXCR4 antibody) or use of anti-PD-1 antibodies have been implicated in subsequent development of severe GVHD. ${ }^{12,13}$ Drug interactions between concomitant medications (azole antifungals, acetaminophen, metronidazole, IDH inhibitors) or recreational drugs (ethanol, marijuana) and commonly used conditioning agents such as busulfan should be considered. ${ }^{14}$ The importance of reviewing all prior therapies, including schedule, duration, and dosage, at the time of evaluation for allo-HCT cannot be overstated.

\section{Disease Status}

Disease remission status and responsiveness to the preceding therapy is a strong predictor of long-term survival after allo-HCT in various hematologic malignancies. ${ }^{6}, 15-19$ It is crucial that prospective patients undergo appropriate diagnostic tests to assess remission status, usually within 1 month of the planned HCT. ${ }^{2}$ These tests are 


\section{Table 1. Selected Indications for Allogeneic HCT}

\begin{tabular}{|c|c|}
\hline Diagnosis & Disease Status/Time \\
\hline \multirow[t]{4}{*}{ AML } & CR1, intermediate risk \\
\hline & CR1, high risk \\
\hline & $\mathrm{CR} 2+$ \\
\hline & Not in remission \\
\hline \multirow[t]{4}{*}{ Acute lymphoblastic leukemia } & CR1, standard risk \\
\hline & CR1, high risk \\
\hline & $\mathrm{CR} 2+$ \\
\hline & Not in remission \\
\hline $\begin{array}{l}\text { Biphenotypic/Mixed- } \\
\text { phenotype } \\
\text { leukemia }\end{array}$ & $\mathrm{CR} 1+$ \\
\hline \multirow[t]{2}{*}{ Chronic myeloid leukemia } & Chronic phase $1, \mathrm{TKI}$ intolerant/refractory \\
\hline & $\begin{array}{l}\text { Chronic phase } \geq 2 \text { or accelerated/blast } \\
\text { phase }\end{array}$ \\
\hline MDS & $\begin{array}{l}\text { Intermediate-2/high risk (early HCT) Low } \\
\text { risk/intermediate-1 (delayed HCT) }\end{array}$ \\
\hline Therapy-related AML/MDS & Untreated, CR1 or beyond \\
\hline $\begin{array}{l}\text { Myelofibrosis \& } \\
\text { myeloproliferative } \\
\text { diseases, including CMML }\end{array}$ & Yes \\
\hline Plasma cell disorders & Relapsed or refractory \\
\hline \multirow[t]{2}{*}{ Hodgkin lymphoma } & Relapsed/Refractory \\
\hline & Relapse after autologous transplant \\
\hline \multirow[t]{2}{*}{ Diffuse large B-cell lymphoma } & Relapsed/Refractory \\
\hline & Relapse after autologous transplant \\
\hline \multirow[t]{2}{*}{ Follicular lymphoma } & Relapsed/Refractory \\
\hline & Relapse after autologous transplant \\
\hline \multirow[t]{2}{*}{ Mantle cell lymphoma } & Relapsed/Refractory \\
\hline & Relapse after autologous transplant \\
\hline \multirow[t]{3}{*}{ Peripheral T-cell lymphoma } & CR1+ \\
\hline & Relapsed/Refractory \\
\hline & Relapse after autologous transplant \\
\hline Cutaneous T-cell lymphoma & Relapsed/Refractory/Advanced \\
\hline \multirow[t]{4}{*}{ Chronic lymphocytic leukemia } & High risk, relapsed/refractory \\
\hline & T-cell prolymphocytic leukemia \\
\hline & B-cell prolymphocytic leukemia \\
\hline & Transformation to high-grade lymphoma \\
\hline
\end{tabular}

(continued)

largely dependent on the underlying diagnosis, but for hematologic malignancies typically include bone marrow biopsies with cytogenetic and molecular assessments, imaging studies, and urine and blood tests (Table 2).

The heterogeneity between disease histology and remission status and its impact on posttransplant outcomes makes data interpretation problematic in studies that include various disease subtypes and status. The disease risk index (DRI) is a validated tool that was

\begin{tabular}{|ll|}
\hline Table 1. Selected Indications for Allogeneic HCT \\
(cont.)
\end{tabular}

Abbreviations: $\mathrm{AML}$, acute myeloid leukemia; $\mathrm{CMML}$, chronic myelomonocytic leukemia; $\mathrm{CR}$, complete remission; $\mathrm{HCT}$, hematopoietic cell transplantation; MDS, myelodysplastic syndromes; SCID, severe combined immunodeficiency; TKI, tyrosine kinase inhibitor.

aldeally in the context of a clinical trial.

bIncluding dyskeratosis congenita, Shwachman-Diamond syndrome, congenital sideroblastic anemia, GATA2 deficiency-associated bone marrow disorder, SAMD9- or SAMDL9-associated disorders, and paroxysmal nocturnal hemoglobinuria.

Data from Majhail NS, Farnia SH, Carpenter PA, et al. Indications for autologous and allogeneic hematopoietic cell transplantation: guidelines from the

American Society for Transplantation and Cellular Therapy (ASTCT). Biol Blood Marrow Transplant 2015;21:1863-1869.

developed using disease-related factors only (diagnosis, remission status, and cytogenetics for AML/MDS) to broadly stratify patients based on disease risk categories. ${ }^{20,21} \mathrm{In}$ one study, DRI assignment into low-, intermediate-, high-, and very high-risk categories in $>13,000$ registry patients undergoing allo-HCT reported 2-year OS rates of $64 \%, 51 \%, 34 \%$, and $24 \%$, respectively. ${ }^{21}$ The DRI of individual patients can be assessed in clinic using an online calculator (available at https://www.cibmtr.org/ ReferenceCenter/Statistical/Tools/Pages/DRI.asp). Although DRI alone is currently not widely used for patient selection, when combined with patient-related factors such Karnofsky performance status (KPS) and HCTcomorbidity index (HCT-CI) (discussed later), these can be invaluable tools for outcomes research and clinical discussions. For specific disease entities such as AML and MDS, prognostic models that predict outcomes and survival have been developed and widely used, which are often considered in the decision-making process. ${ }^{22,23}$ 


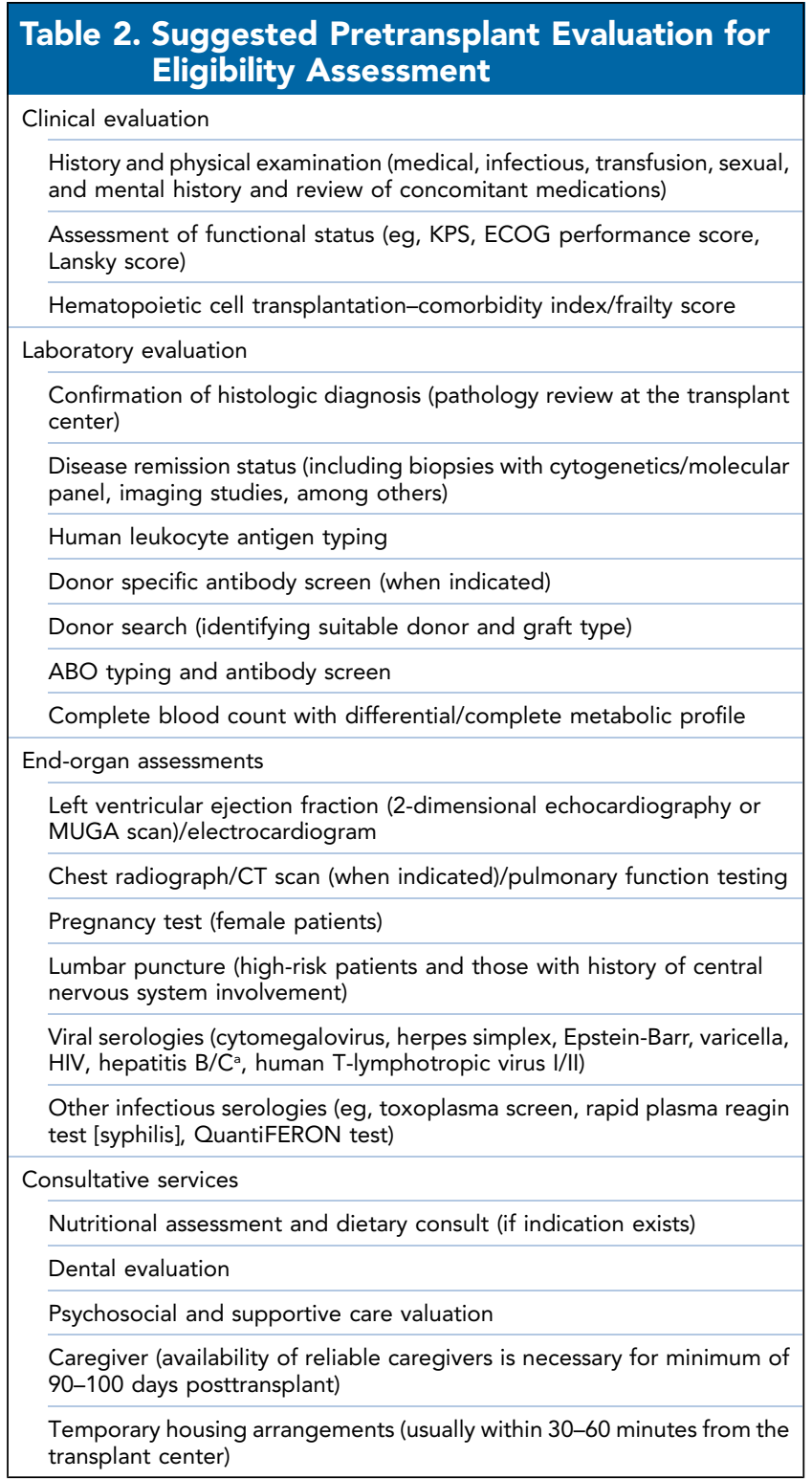

Abbreviation: KPS, Karnofsky performance status; MUGA, multigated acquisition scan.

alf positive, then confirmatory quantitative nucleic acid amplification test is performed

\section{Finding a Donor}

Identifying a suitable donor in a timely fashion is often a rate-limiting step in the consideration of allo-HCT. For a patient with an appropriate indication, initiating human leukocyte antigen (HLA) typing and donor search as soon as possible is recommended. In the absence of a suitable HLA-identical sibling donor (arguably the preferred donor in most centers), a search for a matched unrelated donor (MUD), a mismatched unrelated donor, a haploidentical related donor, or cord blood units should be considered. ${ }^{24-27}$ The search for a MUD can take up to 2 to 3 months, reinforcing the need for early initiation of donor searches, especially in patients with advanced and/or high-risk disease. Recipient screening for donor-specific antibodies is recommended when considering alternative donor sources in order to minimize the risk of primary graft failure. $^{28}$

\section{Patient-Related Factors}

For patients who have an appropriate disease indication and an available suitable donor, the process of physical assessments, rigorous evaluation of end-organ function, and extensive pretransplant counseling should be initiated. Eligibility assessment of patient-related factors, including physiologic, psychological, and socioeconomic considerations, have important implications on posttransplant outcomes. The exact eligibility criteria of patientrelated factors vary widely based institutional practice preferences. Table 3 lists the commonly considered patient variables for allo-HCT eligibility.

\section{Physiologic Factors}

\section{Comorbidities}

Assessment of comorbidities is a vital component of the evaluation of a potential allo-HCT candidate, which includes clinical history, physical examination, laboratory parameters, and other objective tests to determine end-organ function (Table 2). A comprehensive, systemby-system review of pretransplant evaluation is available elsewhere. ${ }^{2,5,29,30}$ Sorror et $\mathrm{al}^{31}$ developed and validated the HCT-CI, in which weighted assignments are given to various comorbidities to create a scoring system that predicted NRM risk following allo-HCT. The HCT-CI has been validated in myeloablative conditioning and RIC allo-HCT and in adult and pediatric patients, and is now widely used by the transplant community. Seventeen comorbidity categories are used for a total HCT-CI score of 0 to 27, depending on the number of concomitant comorbidities in an individual patient (Table 4). In the validation set $(n=346)$, a score of 0 to 2 was associated with a 2 -year NRM of $14 \%$ to $19 \%$ whereas a score of $\geq 3$ resulted in a 2 -year OS of $40 \% .{ }^{31}$ The HCT-CI score is routinely used in clinical practice to estimate a patient's risk of NRM following allograft. Many centers consider switching to RIC regiments (instead of myeloablative conditioning approaches) in young patients with a high HCT-CI score to mitigate risk of NRM. However, it should be noted that no specific score has been set by consensus at which allo-HCT would be contraindicated. Although scoring systems such as HCT-CI, revised pretransplant assessment of mortality (rPAM), EBMT, and combined prognostic scores can be useful for risk stratification, caution is warranted when using them for decision-making in individual patients. ${ }^{32}$ 
Table 3. Suggested Patient and End-Organ Parameters for Allo-HCT Eligibility

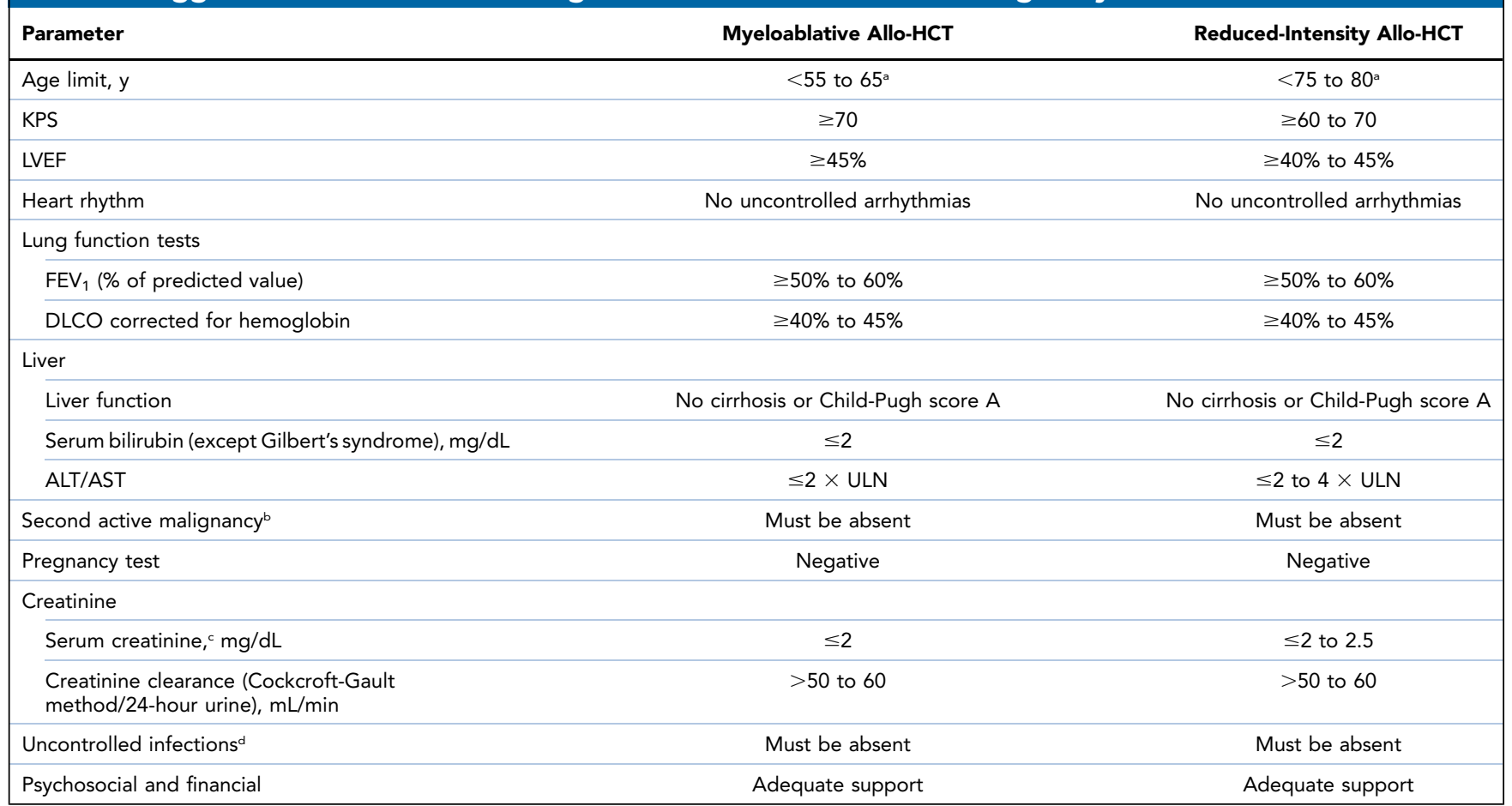

Abbreviations: allo-HCT, allogeneic hematopoietic cell transplantation; ALT, alanine aminotransferase; AST, aspartate aminotransferase; DLCO, diffusion capacity; FEV 1 , force expiratory volume in 1 second; FVC, forced vital capacity; KPS, Karnofsky performance status; LVEF, left ventricular ejection fraction; ULN, upper limit of normal.

aNo absolute cutoff, but largely dependent on institutional practice and insurance approval.

bSecond cancer for which the patient is currently on active therapy and/or survival is likely to be $<5$ years. Indolent malignancies on active surveillance alone may be considered for allo-HCT on an individual basis.

'Except multiple myeloma.

dPatients with HIV infection may be considered if, with appropriate therapy, undetectable viral loads and no evidence of AIDS are noted. Patients with chronic hepatitis $\mathrm{B}$ and $\mathrm{C}$ without active hepatitis may be considered.

\section{Age}

Advanced recipient age alone is no longer considered a contraindication for allo-HCT. The development of RIC/NMAC regimens, better supportive care measures, and infection control have resulted in significant improvements in NRM after allo-HCT, thus broadening the applicability of allo-HCT to include older patients. There has been a significant increase in patients aged $>60$ years undergoing allo-HCT in the past 2 decades; in 2018, 39\% of recipients were aged $\geq 60$ years and $9 \%$ were aged $\geq 70$ years. ${ }^{1}$ McClune et al ${ }^{33}$ reported on the effect of age in patients with AML/MDS undergoing RIC allo-HCT $(n=1,080)$ and noted that unfavorable cytogenetics and performance status, and not age, affected survival. A similar study in older patients with lymphoma (aged $\geq 65$ years) undergoing allo-HCT showed that although age impacted NRM by multivariate analysis, there was no difference in OS when compared with a younger cohort of patients. ${ }^{34}$ Indeed, a patient's physiologic age, rather than their chronologic age, should be considered when deciding eligibility for allo-HCT. The past decade has certainly witnessed an increasing proportion of patients aged $\geq 75$ years undergoing allo-HCT. ${ }^{1,35}$ However, advancing age is often associated with decline in performance levels, frailty, and incipient comorbidities, underscoring the need for rigorous pretransplant assessments and carefully deciding the appropriate allograft strategy. Caution is warranted when considering older individuals for allo-HCT.

\section{Performance Status}

Performance status independently measures the functional ability and health of an individual. Poor performance status at the time of allo-HCT can adversely impact transplant outcomes, including survival. ${ }^{36-38}$ The commonly adopted tool in allo-HCT research and outcome reporting is the KPS, which grades patients in decrements of 10 (or Lansky score in pediatric patients). Most prospective trials in allo-HCT consider a KPS of $<70$ as exclusion criteria for enrollment. ${ }^{2}$ However, it may be emphasized that there is no absolute cutoff point that can be considered exclusionary for consideration of allo-HCT reliably. Although in most institutional practices a KPS of $\geq 60$ to 70 is ideally 
Table 4. HCT-Specific Comorbidity Index ${ }^{31}$

\begin{tabular}{|c|c|c|}
\hline Comorbidity & Explanation & Score \\
\hline Arrhythmia & Atrial fibrillation or flutter, sick sinus syndrome, or ventricular arrhythmias & 1 \\
\hline Cardiac & Coronary artery disease, congestive heart failure, ${ }^{a}$ myocardial infarction, or $\mathrm{EF} \leq 50 \%$ & 1 \\
\hline Inflammatory bowel disease & Crohn disease or ulcerative colitis & 1 \\
\hline Diabetes & Requiring treatment with insulin or oral hypoglycemics & 1 \\
\hline CVD & Transient ischemic attack or cerebrovascular accident & 1 \\
\hline Psychiatric & Depression or anxiety requiring psychiatric consult or treatment & 1 \\
\hline Hepatic, mild & Chronic hepatitis, bilirubin $>$ ULN to $1.5 \times$ ULN, or AST/ALT > ULN to $2.5 \times$ ULN & 1 \\
\hline Obesity & Body mass index $>35 \mathrm{~kg} / \mathrm{m}^{2}$ & 1 \\
\hline Infection & Requiring treatment after day 0 & 1 \\
\hline Rheumatologic & SLE, rheumatoid arthritis, polymyositis, mixed CTD, or polymyalgia rheumatica & 2 \\
\hline Peptic ulcer & Requiring treatment & 2 \\
\hline Moderate/Severe renal & Serum creatinine $>2 \mathrm{mg} / \mathrm{dL}$, on dialysis, or prior renal transplantation & 2 \\
\hline Moderate pulmonary & DLCO and/or FEV $166 \%-80 \%$ or dyspnea with activity & 2 \\
\hline Prior solid tumor & Treated at any time point in the patient's history, excluding nonmelanoma skin cancer & 3 \\
\hline Heart valve disease & All, except mitral valve prolapse & 3 \\
\hline Severe pulmonary & DLCO and/or $\mathrm{FEV}_{1} \leq 65 \%$ or dyspnea at rest or requiring oxygen & 3 \\
\hline Moderate/Severe hepatic & Liver cirrhosis, bilirubin $>1.5 \times$ ULN, or AST/ALT $>2.5 \times$ ULN & 3 \\
\hline
\end{tabular}

Abbreviations: ALT, alanine aminotransferase; AST, aspartate aminotransferase; CTD, connective tissue disease; CVD, cerebrovascular disease; DLCO, diffusion capacity of carbon monoxide; $E F$, ejection fraction; $F_{E V}$, force expiratory volume in 1 second; HCT, hematopoietic cell transplantation; SLE, systemic lupus erythematosus; ULN, upper limit of normal.

aOne or more vessel: coronary artery stenosis requiring medical treatment, stent, or bypass graft.

required to consider allo-HCT, each patient should be evaluated and considered on a case-by-case basis in a multidisciplinary setting.

\section{Frailty}

Frailty is defined as a state of decreased physiologic reserve associated with vulnerability to disability and death, is usually associated with advancing age, and is an aggregate of cognition, mobility, function, and comorbidity. ${ }^{39-41}$ Frailty adversely impacts tolerance and response to cancer therapy, and mortality and frailty valuations are now recommended by professional organizations to be included in the routine care of geriatric patients with cancer. $^{41,42}$ In one study, investigators prospectively evaluated grip strength and gait speed in 448 patients aged $\geq 75$ years with hematologic malignancies and found that every $0.1 \mathrm{~m} / \mathrm{sec}$ decrease in gait speed was associated with increased mortality and higher unplanned hospitalizations and emergency department visits, whereas every 5-kg decrease in grip strength was associated with worse survival. ${ }^{40}$ Currently, no validated tool for frailty/geriatric assessments exist specifically for patients being considered for allo-HCT. At West Virginia University, in collaboration with the physical therapy service, the 2-minute walk, isometric handgrip exercise, Five Times Sit-to-Stand, and Timed $\mathrm{Up}$ and Go tests are performed during the pretransplant evaluation. ${ }^{43}$ A multidisciplinary approach to assess and optimize the treatment plan based on geriatric assessments may improve posttransplant outcomes. ${ }^{44}$ The ongoing BMT CTN 1704 study Composite Health Assessment Risk Model (CHARM) for Older Adults (ClinicalTrials.gov identifier: NCT03992352) will prospectively apply comorbidity index, geriatric assessment, and biomarkers such as albumin and C-reactive protein to NRM and other transplant outcomes after allo-HCT in patients aged $\geq 60$ years. It should be noted that frailty assessments in the pretransplant evaluation remain an area of emerging interest and active research and are not used routinely in transplant evaluation.

\section{Psychologic Factors}

Careful assessment of a patient's psychological health and underlying psychiatric conditions, such as depression, anxiety, and bipolar disorder, is necessary during pretransplant evaluation. Addressing patients' concerns and anxiety and other psychological issues is of paramount importance before committing them to intensive and life-altering therapy such as allo-HCT. ${ }^{45}$ In fact, psychosocial factors such as relationship status and somatic symptoms have been associated with transplant outcomes. ${ }^{46}$ In our experience, prior history of noncompliance, lack of motivation for self-care, active 
alcohol/substance abuse, and untreated or uncontrolled psychiatric illnesses are warning signs and often counterproductive in the posttransplant setting and should be carefully evaluated. In our centers, all potential transplant patients are evaluated at least once in the oncology supportive care service, with further follow-up or referral to psychiatric oncology services as appropriate.

\section{Socioeconomic and Ethnic Factors}

Socioeconomic circumstances of a transplant candidate play an important role in determining their access to transplantation and can impact procedure outcomes. Most transplant centers require a dedicated caregiver to be available in the first 100 days of transplant care. Poor caregiver structure and single relationship status have previously been shown to result in poor HCT outcomes and lower quality-of-life indices. ${ }^{46,47}$ Undergoing allo-HCT will invariably result in loss of income for a prolonged period, with resultant financial strain on patients and their families. ${ }^{48}$ Low socioeconomic status, absence of private insurance, lack of accessibility to transplant care, and poor health literacy are hindrances to successful transplantation..$^{49,50}$ Financial coordinators and social workers play an indelible role in navigating matters such as insurance coverage and short-term disability, childcare, transportation, and temporary housing options-details that significantly affect a patient's ability to undergo transplantation, mental health, and compliance with posttransplant care.

Racial and ethnic disparities in the use of and access to allo-HCT and in posttransplant outcomes have been noted. Data suggest that black patients are less likely to undergo allo-HCT compared with white patients. ${ }^{49} \mathrm{~A}$ registry study in $>6,000$ patients undergoing allo-HCT noted inferior OS in black patients compared with white patients, and a higher NRM was observed in Hispanic and black patients. ${ }^{50}$ It is likely that socioeconomic barriers rather than ethnicity is the underlying cause for this disparity. Thus, ethnicity alone should not affect the decision to consider allo-HCT for an individual, but the influence and potential causes of these disparities must be carefully weighed (eg, socioeconomic background, health literacy, genetic variations) before the final decision is made. ${ }^{29}$

\section{Integrating Eligibility Assessment Into Clinical Practice}

Acknowledging the lack of randomized data or consensus recommendations, this review provides some perspectives on how to integrate the aforementioned eligibility assessments into clinical practice. It should be noted that several of these parameters may be concomitantly evaluated in the interest of time. First, they establish whether allo-HCT is a suitable treatment strategy for the underlying diagnosis, typically following the ASTCT position paper on allo-HCT indications. ${ }^{6}$ Integrating disease status into clinical decision-making before transplant is critical for patient outcomes. Patients with uncontrolled malignancies (eg, refractory acute leukemia, rapidly progressing lymphomas) are unlikely to benefit from allo-HCT and warrant better disease control before embarking on transplantation. Second, for patients in whom allo-HCT is indicated, patient-related factors should be assessed carefully, including review of comorbidities, performance status, frailty indicators, and patient age, to establish candidacy. Unfortunately, no evidence-based cutoffs are currently available that represent absolute contraindications for allo-HCT. A 75-year-old woman with an excellent KPS and no comorbidities may be considered for RIC transplant, whereas a 45-year-old woman with a KPS of 50 and multiple comorbidities may not be an ideal candidate. HCT-CI is a highly valuable tool, but no specific score can be used to determine whether to move forward with allografting. For example, a patient with an HCT-CI score of 6 (diabetes, obesity, depression, and remote history of treated breast cancer) may be offered an allograft, whereas a patient with HCT-CI score of 3 but with an ejection fraction of $10 \%$ might not. Third, psychologic and social parameters must be considered. For example, in the authors' centers, patients with uncontrolled psychiatric illness or no available caregiver are generally (but not always) deemed ineligible for transplant. Patients with advanced organ dysfunction are also not ideal transplant candidates (eg, unstable angina, uncontrolled heart failure, low cardiac or pulmonary reserve, advanced renal insufficiency).

Once the decision is made to offer allogeneic HCT as a treatment option, several factors, such as disease status, prior therapies, and HCT-CI score, can be used to optimize the transplant strategy, including donor type, conditioning intensity, and supportive care. For example, (1) in the authors' practices, RIC/NMAC approaches are favored for any patients with lymphoma or myeloma, elderly individuals (age $>60-65$ years), and those with an HCT-CI score $>4$; (2) myeloablative conditioning should be considered for patients with AML, especially those with genomic evidence of minimal residual disease ${ }^{51}$; (3) patients who are identified as high risk for VOD (https:// www.cibmtr.org/ReferenceCenter/Statistical/Tools/Pages/ VOD.aspx) should be offered conditioning regimens (eg, total body irradiation-free, lower doses of busulfan) and GVHD prophylaxis that could potentially lower the risk; and (4) infectious history and diagnostic workup can help determine appropriate prevention strategies, such as use of antimold or anticytomegalovirus agents or monitoring (in the appropriate setting). A vital tool available to transplant centers is the Center for International 
Blood \& Marrow Transplant Research's Survival Calculator that predicts 1-year survival for patients who have undergone allo-HCT based on the 3-year transplant center-specific survival dataset (https://www.cibmtr.org/ ReferenceCenter/Statistical/Tools/Pages/index.aspx). In the authors' centers, the benefits and disadvantages of allo-HCT are carefully discussed with patients who are otherwise eligible but who have poor 1-year predicted survival (generally $<25 \%$ to $30 \%$ ) probability. The authors recommend integrating the calculator into the initial consideration and education of a prospective transplant patient.

\section{Conclusions}

The pretransplant eligibility evaluation is a complex, multifaceted, and multidisciplinary process and involves assessments of disease-related factors (diagnosis, remission status, DRI) and patient-related factors, including physiologic (age, KPS, HCT-CI, frailty score), psychosocial, and economic parameters. No uniform consensus or strict guidelines exist regarding eligibility criteria, and the final decision is largely at the discretion of the transplant center and team. Given the paucity of data, whenever possible, enrollment in carefully chosen clinical trials is most appropriate. Careful consideration should be given to the results of pretransplant eligibility evaluation, exhaustive review of available literature to support allo-HCT versus other treatment options, and frank discussion with the patient and family to ascertain their perceptions and expectations before committing a patient to alloHCT.

\section{Acknowledgments}

The authors with to express their gratitude to Dr. Navneet Majhail, Director, Blood \& Marrow Transplant Program, Cleveland Clinic Taussig Cancer Institute, and Dr. Javier Bolaños-Mead, Clinical Director, Adult Bone Marrow Transplant Program, The Sidney Kimmel Comprehensive Cancer Center at Johns Hopkins, for their valuable expertise, input, and critique.

Submitted November 27, 2019; accepted for publication March 10, 2020.

Disclosures: Dr. Perales has disclosed that he has received support from $\mathrm{NIH}$ (award P01 CA23766) and NIH/NCl Cancer Center Support Grant (P30 CA008748); honoraria from Abbvie, Bellicum, Celgene, Bristol-Myers Squibb, Incyte, Merck, Novartis, Nektar Therapeutics, Omeros, and Takeda; serves as a scientific advisor for Cidara Therapeutics, Servier, and Medigene, MolMed, and Nexlmmune; and received grant/research support from Incyte, Kite/Gilead and Miltenyi Biotec. Dr. Hamadani has disclosed that he receives grant/research support from Takeda Pharmaceutical Company, Otsuka Pharmaceutical, Spectrum Pharmaceuticals, Astellas Pharma, Janssen R\&D, Celgene Corporation, Merck, Medlmmune, Seattle Genetics, and Millennium Pharmaceuticals; serves as a consultant for Medlmmune LLC, Janssen R \&D, Incyte Corporation, ADC Therapeutics, Cellerant Therapeutics, Celgene Corporation, Pharmacyclics \& DOVA Oncology, Magenta Therapeutics, Omeros, and Verastem; and serves as a scientific advisor for Sanofi Genzyme, and AstraZeneca. Dr. Kanate has disclosed that he has no financial interests, arrangements, affiliations, or commercial interests with the manufacturers of any products discussed in this article or their competitors

Correspondence: Mehdi Hamadani, MD, Department of Medicine, Center for International Blood and Marrow Transplant Research, Medical College of Wisconsin, 9200 West Wisconsin Avenue, Suite C5500, Milwaukee, WI 53226. Email: mhamadani@mcw.edu

\section{References}

1. D'Souza A, Fretham C. Current uses and outcomes of hematopoietic cell transplantation (HCT): CIBMTR summary slides, 2019. Accessed March 16, 2020. Available at: https://www.cibmtr.org

2. Scott B, Sandmaier B. The evaluation and counseling of candidates for hematopoietic cell transplantation. In: Forman JS, Negrin RS, Antin JH, Appelbaum FR, eds. Thomas' Hematopoietic Cell Transplantation, 5th ed. Boston, MA: Blackwell Publishing; 2016:349-365.

3. McDonald GB, Sandmaier BM, Mielcarek M, et al. Survival, nonrelapse mortality, and relapse-related mortality after allogeneic hematopoietic cell transplantation: comparing 2003-2007 versus 2013-2017 cohorts [published online January 21, 2020]. Ann Intern Med, doi: https://doi.org/ 10.7326/M19-2936

4. Timing Impact on Outcomes. Accessed November 1, 2019. Available at: https://bethematchclinical.org/transplant-indications-and-outcomes/ additional-outcomes/timing-impact-on-outcomes

5. Hamadani M, Craig M, Awan FT, et al. How we approach patient evaluation for hematopoietic stem cell transplantation. Bone Marrow Transplant 2010;45:1259-1268.

6. Majhail NS, Farnia SH, Carpenter PA, et al. Indications for autologous and allogeneic hematopoietic cell transplantation: guidelines from the American Society for Blood and Marrow Transplantation. Biol Blood Marrow Transplant 2015;21:1863-1869.

7. Perales MA, Ceberio I, Armand P, et al. Role of cytotoxic therapy with hematopoietic cell transplantation in the treatment of Hodgkin lymphoma: guidelines from the American Society for Blood and Marrow Transplantation. Biol Blood Marrow Transplant 2015; 21:971-983

8. de Witte $T$, Bowen D, Robin M, et al. Allogeneic hematopoietic stem cell transplantation for MDS and CMML: recommendations from an international expert panel. Blood 2017;129:1753-1762.
9. Freytes CO, Zhang MJ, Carreras J, et al. Outcome of lower-intensity allogeneic transplantation in non-Hodgkin lymphoma after autologous transplantation failure. Biol Blood Marrow Transplant 2012;18: 1255-1264.

10. Baron J, Wang ES. Gemtuzumab ozogamicin for the treatment of acute myeloid leukemia. Expert Rev Clin Pharmacol 2018;11:549-559.

11. Kantarjian HM, DeAngelo DJ, Stelljes M, et al. Inotuzumab ozogamicin versus standard of care in relapsed or refractory acute lymphoblastic leukemia: final report and long-term survival follow-up from the randomized, phase 3 INO-VATE study. Cancer 2019;125: 2474-2487.

12. Sugio T, Kato K, Aoki T, et al. Mogamulizumab treatment prior to allogeneic hematopoietic stem cell transplantation induces severe acute graft-versus-host disease. Biol Blood Marrow Transplant 2016;22: 1608-1614.

13. Merryman RW, Kim HT, Zinzani PL, et al. Safety and efficacy of allogeneic hematopoietic stem cell transplant after PD-1 blockade in relapsed/ refractory lymphoma. Blood 2017;129:1380-1388.

14. Myers AL, Kawedia JD, Champlin RE, et al. Clarifying busulfan metabolism and drug interactions to support new therapeutic drug monitoring strategies: a comprehensive review. Expert Opin Drug Metab Toxicol 2017;13:901-923.

15. Warlick ED, Cioc A, Defor T, et al. Allogeneic stem cell transplantation for adults with myelodysplastic syndromes: importance of pretransplant disease burden. Biol Blood Marrow Transplant 2009;15:30-38.

16. Sarina B, Castagna L, Farina L, et al. Allogeneic transplantation improves the overall and progression-free survival of Hodgkin lymphoma patients relapsing after autologous transplantation: a retrospective study based on the time of HLA typing and donor availability. Blood 2010; 115:3671-3677 
17. Fenske TS, Ahn KW, Graff TM, et al. Allogeneic transplantation provides durable remission in a subset of DLBCL patients relapsing after autologous transplantation. Br J Haematol 2016;174:235-248.

18. Weisdorf DJ, Millard HR, Horowitz MM, et al. Allogeneic transplantation for advanced acute myeloid leukemia: the value of complete remission. Cancer 2017;123:2025-2034.

19. Koreth J, Schlenk R, Kopecky KJ, et al. Allogeneic stem cell transplantation for acute myeloid leukemia in first complete remission: systematic review and meta-analysis of prospective clinical trials. JAMA 2009;301:2349-2361.

20. Armand $\mathrm{P}$, Gibson $\mathrm{CJ}$, Cutler $\mathrm{C}$, et al. A disease risk index for patients undergoing allogeneic stem cell transplantation. Blood 2012;120: 905-913.

21. Armand $P$, Kim HT, Logan BR, et al. Validation and refinement of the Disease Risk Index for allogeneic stem cell transplantation. Blood 2014; 123:3664-3671.

22. Greenberg PL, Tuechler H, Schanz J, et al. Revised international prognostic scoring system for myelodysplastic syndromes. Blood 2012;120 2454-2465.

23. Döhner H, Estey E, Grimwade D, et al. Diagnosis and management of AML in adults: 2017 ELN recommendations from an international expert panel. Blood 2017;129:424-447.

24. Saber W, Opie S, Rizzo JD, et al. Outcomes after matched unrelated donor versus identical sibling hematopoietic cell transplantation in adults with acute myelogenous leukemia. Blood 2012;119:3908-3916.

25. Brunstein CG, Fuchs EJ, Carter SL, et al. Alternative donor transplantation after reduced intensity conditioning: results of parallel phase 2 trials using partially HLA-mismatched related bone marrow or unrelated double umbilical cord blood grafts. Blood 2011;118:282-288.

26. Kanate AS, Mussetti A, Kharfan-Dabaja MA, et al. Reduced-intensity transplantation for lymphomas using haploidentical related donors vs HLA-matched unrelated donors. Blood 2016;127:938-947.

27. Ghosh N, Karmali R, Rocha V, et al. Reduced-intensity transplantation for lymphomas using haploidentical related donors versus HLA-matched sibling donors: a Center for International Blood and Marrow Transplant Research analysis. J Clin Oncol 2016;34:3141-3149.

28. Dehn J, Spellman S, Hurley CK, et al. Selection of unrelated donors and cord blood units for hematopoietic cell transplantation: guidelines from the NMDP/CIBMTR. Blood 2019;134:924-934.

29. Deeg HJ, Sandmaier B. Determining eligibility for allogeneic hematopoietic cell transplantation. Accessed November 5, 2019. Available at: https://www.uptodate.com/contents/determining-eligibility-for-allogeneic-hematopoietic-cell-transplantation/print

30. Patient Eligibility for HCT. Accessed November 5, 2019. Available at: https://bethematchclinical.org/transplant-indications-and-outcomes/ eligibility

31. Sorror ML, Maris MB, Storb R, et al. Hematopoietic cell transplantation (HCT)-specific comorbidity index: a new tool for risk assessment before allogeneic HCT. Blood 2005;106:2912-2919.

32. Shouval R, Fein JA, Shouval A, et al. External validation and comparison of multiple prognostic scores in allogeneic hematopoietic stem cell transplantation. Blood Adv 2019;3:1881-1890.

33. McClune BL, Weisdorf DJ, Pedersen TL, et al. Effect of age on outcome of reduced-intensity hematopoietic cell transplantation for older patients with acute myeloid leukemia in first complete remission or with myelodysplastic syndrome. J Clin Oncol 2010;28:1878-1887.

34. Shah NN, Ahn KW, Litovich C, et al. Outcomes of Medicare-age eligible NHL patients receiving RIC allogeneic transplantation: a CIBMTR analysis. Blood Adv 2018;2:933-940.
35. Muffly L, Pasquini MC, Martens M, et al. Increasing use of allogeneic hematopoietic cell transplantation in patients aged 70 years and older in the United States. Blood 2017;130:1156-1164.

36. Sorror M, Storer B, Sandmaier BM, et al. Hematopoietic cell transplantation-comorbidity index and Karnofsky performance status are independent predictors of morbidity and mortality after allogeneic nonmyeloablative hematopoietic cell transplantation. Cancer 2008;112 1992-2001

37. Artz AS, Pollyea DA, Kocherginsky M, et al. Performance status and comorbidity predict transplant-related mortality after allogeneic hematopoietic cell transplantation. Biol Blood Marrow Transplant 2006;12: 954-964.

38. Sayer HG, Kröger M, Beyer J, et al. Reduced intensity conditioning for allogeneic hematopoietic stem cell transplantation in patients with acute myeloid leukemia: disease status by marrow blasts is the strongest prognostic factor. Bone Marrow Transplant 2003;31:1089-1095.

39. Wildiers $\mathrm{H}$, Heeren $\mathrm{P}$, Puts $\mathrm{M}$, et al. International Society of Geriatric Oncology consensus on geriatric assessment in older patients with cancer. $\mathrm{J}$ Clin Oncol 2014;32:2595-2603.

40. Liu MA, DuMontier C, Murillo A, et al. Gait speed, grip strength, and clinical outcomes in older patients with hematologic malignancies. Blood 2019;134:374-382.

41. Abel GA, Klepin HD. Frailty and the management of hematologic malignancies. Blood 2018;131:515-524.

42. Mohile SG, Dale W, Somerfield MR, et al. Practical assessment and management of vulnerabilities in older patients receiving chemotherapy: ASCO guideline for geriatric oncology. J Clin Oncol 2018;36 2326-2347

43. Caveney J, Kanate AS, Hobbs G, Swisher A. Relationship of physical function to outcomes in autologous hematopoietic stem cell transplantation [abstract]. J Clin Oncol 2019;37(Suppl):Abstract 116.

44. Derman BA, Kordas K, Ridgeway J, et al. Results from a multidisciplinary clinic guided by geriatric assessment before stem cell transplantation in older adults. Blood Adv 2019;3:3488-3498

45. Andorsky DJ, Loberiza FR, Lee SJ. Pre-transplantation physical and mental functioning is strongly associated with self-reported recovery from stem cell transplantation. Bone Marrow Transplant 2006;37:889-895.

46. Pillay B, Lee SJ, Katona L, et al. Psychosocial factors predicting survival after allogeneic stem cell transplant. Support Care Cancer 2014;22: 2547-2555

47. Molassiotis A, van den Akker OBA, Boughton BJ. Perceived social support, family environment and psychosocial recovery in bone marrow transplant long-term survivors. Soc Sci Med 1997;44:317-325.

48. Meehan KR, Fitzmaurice $T$, Root $L$, et al. The financial requirements and time commitments of caregivers for autologous stem cell transplant recipients. J Support Oncol 2006;4:187-190.

49. Majhail NS, Omondi NA, Denzen E, et al. Access to hematopoietic cell transplantation in the United States. Biol Blood Marrow Transplant 2010 16:1070-1075.

50. Baker KS, Davies SM, Majhail NS, et al. Race and socioeconomic status influence outcomes of unrelated donor hematopoietic cell transplantation. Biol Blood Marrow Transplant 2009;15:1543-1554.

51. Hourigan CS, Dillon LW, Gui G, et al. Impact of conditioning intensity of allogeneic transplantation for acute myeloid leukemia with genomic evidence of residual disease [published online December 20, 2019]. J Clin Oncol, doi: 10.1200/JCO.19.03011 